\title{
New developments in the treatment of hyperammonemia: emerging use of carglumic acid
}

This article was published in the following Dove Press journal:

International Journal of General Medicine

6 January 2011

Number of times this article has been viewed

\section{Marta Daniotti' \\ Giancarlo la Marca \\ Patrizio Fiorini' \\ Luca Filippi'}

'Neonatal Intensive Care Unit, Department of Perinatal Medicine, “A. Meyer" University Children's Hospital, Florence, Italy; ${ }^{2}$ Mass Spectrometry, Clinical Chemistry and Pharmacology Laboratory, Neuroscience Department,

“A. Meyer" University Children's Hospital, Florence, Italy
Correspondence: Luca Filippi Neonatal Intensive Care Unit, Department of Perinatal Medicine, "A. Meyer" University Children's Hospital,Viale Pieraccini, 24 I-50I 39 Florence, Italy $\mathrm{Tel}+39-55-5662434$

Fax +39-55-5662400

Email I.filippi@meyer.it
Abstract: Hyperammonemia is a true neonatal emergency with high toxicity for the central nervous system and developmental delay. The causes of neonatal hyperammonemia are genetic defects of urea cycle enzymes, organic acidemias, lysinuric protein intolerance, hyperammonemiahyperornithinemia-homocitrullinemia syndrome, transient hyperammonemia of the newborn, and congenital hyperinsulinism with hyperammonemia. In some of these conditions the high blood ammonia levels are due to the reduction of $\mathrm{N}$-acetylglutamate, an essential cofactor necessary for the function of the urea cycle, or to the reduction of carbamoyl-phosphate synthase-I activity. In these cases, N-carbamylglutamate (carglumic acid) can be administered together with the conventional therapy. Carglumic acid is an analog of $\mathrm{N}$-acetylglutamate that has a direct action on carbamoyl-phosphate synthase-I. Its effects are reactivation of the urea cycle and reduction of plasma ammonia levels. As a consequence it improves the traditional treatment, avoiding the need of hemodialysis and peritoneal dialysis. In this review we evaluate the possible field of application of carglumic acid and its effectiveness and safety.

Keywords: hyperammonemia, N-carbamylglutamate, carglumic acid, urea cycle disorder, metabolic disorders

\section{Introduction}

In humans catabolism of proteins and other nitrogen-containing molecules results in the production of nitrogen. Waste nitrogen is excreted largely as urea with smaller amounts as creatinine, ammonium ion, amino acids, and other nitrogenous compounds. In the brain, ammonia is an important product of the metabolism of the neurotransmitters glutamate and aspartate and of the neurotransmitter monoamines. It is also the product of the synthesis of glutamate from glutamine in nerve endings. At least some of the glutamate released from the nerve endings is neutralized via uptake by the astrocytes where it is combined with ammonia to form the electrophysiologically inactive molecule glutamine. Despite its central importance in metabolism, high concentrations of ammonia are generally toxic, particularly to the central nervous system, and the compound must be maintained at relatively low levels in the tissues.

Many inborn errors of metabolism cause increases in blood ammonia levels. Hyperammonemia is a condition that can cause neurological complications, coma, and even death. Indeed, high concentrations of ammonia may partially saturate the "enzymatic detoxifier" of the astrocytes, impeding the brain's capacity for selfprotection, which contributes to the neurological dysfunction. Therefore, high levels of ammonia are a real emergency and should be treated promptly. First-line treatment 
consists of reducing catabolism and promoting anabolism, by a protein-restriction diet and substituting an oral or intravenous high energy source, such as glucose infusion, and parenteral lipids administration after exclusion of fatty acid oxidation disorder. Because the kidneys clear ammonia poorly, its removal from the body must be expedited by formation of compounds with high renal clearance. Ammoniascavenging drugs (sodium benzoate, sodium phenylbutyrate, and arginine hydrochloride) are at present considered the first-line drugs for the treatment of neonatal hyperammonemia. However, if the foregoing therapies fail to produce any appreciable change in blood ammonia level within a few hours, the neonate should be transferred without delay to a center with hemodialysis capacity, and continuous venovenous hemofiltration, in particular, should be performed.

The administration of $\mathrm{N}$-carbamylglutamate (carglumic acid, NCG) has been reported to be of benefit in several different conditions presenting with hyperammonemia. ${ }^{1}$ $\mathrm{NCG}$ is a synthetic analog of N-acetylglutamate (NAG) that activates carbamoyl-phosphate synthase I (CPS-I), ${ }^{2}$ the enzyme of the first and rate-limiting step of the urea cycle stimulating ureagenesis ${ }^{3}$ (Figure 1). NCG is able to enter into the mitochondria and is not inactivated by cytosolic acylases. ${ }^{4}$ For this reason it is an optimal pharmacological substitute; on the contrary, the natural NAG is hydrolyzed in vivo by acylamino acid acylase. ${ }^{5}$

In this review we evaluate the field of application and the possibility for the treatment of hyperammonemia with NCG.

\section{$\mathbf{N}$-carbamylglutamate in urea cycle disorders}

The urea cycle represents the principal mechanism of ammonia detoxification. Five enzymes are required for ureagenesis: CPS-I, ornithine transcarbamylase, argininosuccinate synthase, argininosuccinate lyase, and arginase. The urea cycle is also regulated by NAG, an essential cofactor necessary for the function of CPS-I. NAG is produced in the mitochondrial matrix from glutamate and acetyl coenzyme A by N-acetylglutamate synthase (NAGS). Deficiency in any one of these enzymes

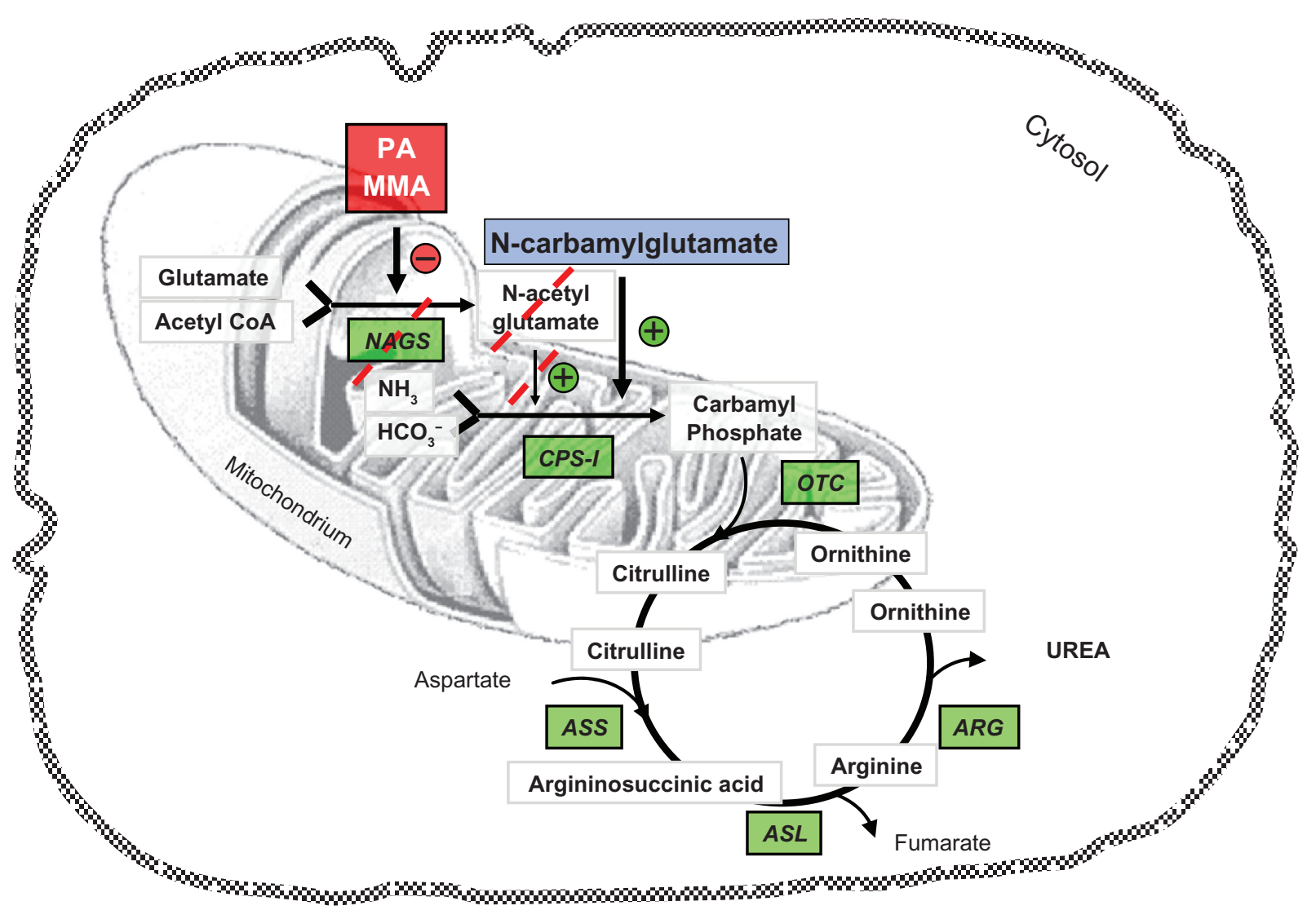

Figure I Schematic representation of the urea cycle and related pathways.

Abbreviations: ASS, argininosuccinate synthase; ASL, argininosuccinate lyase; ARG, arginase; CPS-I, carbamoyl-phosphate synthase I; MMA, methylmalonic acidemia; NAGS, $\mathrm{N}$-acetylglutamate synthase; OTC, ornithine transcarbamylase; PA, propionic acidemia. 
causes a urea cycle disorder which results in ammonia accumulation. ${ }^{6}$

\section{$\mathrm{N}$-acetylglutamate synthase deficiency}

A NAGS deficiency is a rare, autosomal recessive urea cycle disorder, and was described for the first time by Bachmann and colleagues in $1981 .^{7}$ In this congenital error there is a wide range of severity of symptoms, as well as in the age of onset. Most frequently, the onset occurs in the early neonatal period with the signs and symptoms of hyperammonemia: poorly fed, vomiting, and neurological alteration such as hypotonia, abnormal movements of limbs, irritability, generalized seizures, afterward lethargy, and coma. Late forms may present with acute attacks of hyperammonemia in a previously healthy individual or with chronic neurological, gastrointestinal, and psychiatric clinical signs. The symptoms could be chronic nausea, vomiting, ataxia, confusion, and irritability, and in acute cases could lead to coma. The laboratory findings show an increase in blood levels of ammonia and markedly elevated concentrations of glutamine and alanine. In contrast to most of the other urea cycle disorders, plasma citrulline and urinary orotic acid are normal as well as urine organic acids and blood acylcarnitines. These clinical and biochemical features are present both in NAGS and CPS-I deficiencies. The diagnosis could be confirmed either with determination of NAGS and CPS-I activity in liver tissue in presence of arginine or by molecular diagnostic analysis. ${ }^{8}$ The majority of early forms have $<5 \%$ NAGS residual activity and often have frameshift or nonsense mutations. Indeed, the late form presentation is associated with partial NAGS deficiency and hypomorphic alleles with single amino acid substitutions. ${ }^{8-11}$ The treatment of acute hyperammonemia consists of an intravenous administration of adequate calories, fluids, and electrolytes and in a restriction of protein intake but providing a mixture of essential amino acids. NCG is a specific treatment of hyperammonemia due to NAGS deficiency and should be administered as early possible when it is suspected. ${ }^{12}$ Also sodium benzoate (200-500 mg/kg/24 h), sodium phenylacetate $(250-500 \mathrm{mg} / \mathrm{kg} / 24 \mathrm{~h})$, and arginine (200-600 mg/kg/24 h) should be considered. In case of failure of the above treatment, peritoneal dialysis must be initiated. In 1995, Guffon et al described a case of a newborn with NAGS deficiency treated by NCG; this treatment was initiated on the 25 th day of life with a total dose of $80-100 \mathrm{mg} / \mathrm{kg} /$ day, with optimal results. A 1-year follow-up showed an infant with normal somatic and neurological development and a good metabolic balance. ${ }^{13}$
In the following years, 3 other cases, 1 neonatal form and 2 cases of partial NAGS deficiency, were described. ${ }^{14-16}$ NCG was administered (60-100 mg/kg/day in 4 single doses) and a decrease of blood ammonia was observed: this enabled an increase in protein intake. In 2004, Caldovic et al ${ }^{17}$ studied how the incorporation of an isotopic label from ammonium chloride into urea increased after the treatment with NAG. This study provided strong evidence that NCG therapy restores ureagenesis in NAGS deficiency. Later, in 2008, Tuchman et al presented a stable isotope ${ }^{13} \mathrm{C}$ tracer method to measure ureagenesis and evaluate the effect of NCG in humans. The results of these investigations showed the reduction of plasma ammonia and glutamine levels and confirmed that NCG could be an effective treatment for inherited and secondary NAGS deficiency. ${ }^{18}$ Nordenström et al ${ }^{19}$ and Gessler et $\mathrm{al}^{12}$ reported other clinical cases of NAGS deficiency and concluded that the immediate treatment of hyperammonemia with NCG entails a favorable long-term outcome.

Currently NCG is approved in the European Union and the United States for the treatment of hyperammonemia due to NAGS deficiency. The initial daily dose should be $100 \mathrm{mg} / \mathrm{kg}$, up to $250 \mathrm{mg} / \mathrm{kg}$ if necessary. It should then be adjusted individually in order to maintain normal ammonia plasma levels.

\section{Carbamoyl-phosphate synthase I deficiency}

The therapy with NAG in the kinetic variant or partial CPS-I deficiency has been discussed by O'Conner et al in $1985 .^{2}$ In 1996 Kuchler et $\mathrm{al}^{20}$ administered NCG in an infant on suspicion of a NAGS deficiency. After the treatment, the levels of ammonia and glutamine decreased to the normal range. The results of liver biopsy showed a normal activity of NAGS and a decrement of activity of CPS-I. The authors concluded that NCG stimulates the remaining CPS-I and increases ureagenesis, for this reason NCG might be used in patients with partial CPS-I deficiency.

\section{$\mathbf{N}$-carbamylglutamate in organic acidemias}

Neonatal hyperammonemia can be caused by some organic acidemias. Since the 1970s, hyperammonemia has been known to be a complication of propionic acidemia (PA) and methylmalonic acidemia (MMA), due to an inhibition of carbamoyl-phosphate synthesis, the first step of the urea 
cycle. In fact, in such disorders, propionyl-CoA accumulates and inhibits NAGS. The reduced synthesis of NAG, the natural stimulator of CPS-I, hinders the conversion of ammonia to urea in the liver. ${ }^{21}$

\section{Propionic acidemia}

Propionic acid is an intermediate metabolite of branched-chain amino acids (valine, theonine, methionine), odd-chain fatty acids, and cholesterol degradation. Propionic acid is carboxylated to methylmalonic acid by a mitochondrial biotin-dependent enzyme: propionyl CoA carboxylase. It is composed of 2 subunits, $\alpha$ and $\beta$; the subunit $\alpha$ binds biotin. A failure of this enzyme determines the PA (MIM 606054). This inborn disease is inherited as an autosomal recessive trait. The genes involved are located in the following chromosomal regions $13 q 32$ and 3q21-q22, and many mutations have been identified in different patients. Two forms are described based on the clinical manifestations: a severe form in which symptoms develop during the first days of life, and a milder form in which a patient may have an episode of severe ketoacidosis after a healthy period. The clinical findings are poor feeding, vomiting, dehydration, hypotonia, lethargy, severe ketoacidosis with rapid progress to coma, and death. Laboratory findings during the acute attack are a severe metabolic acidosis, hypoglycemia, and hyperammonemia. Propionic acid and methylcitric acid are markedly elevated in the blood and urine of infants with PA. To reach a definite diagnosis, a measurement of the enzyme activity in leukocytes or cultured fibroblasts is required. The treatment consists of hydration, correction of acidosis, with intravenous glucose, insulin, carnitine, sodium benzoate, and vitamin therapy, associated with a hypercaloric protein-free diet. Gebhardt et al described 2 patients with PA and hyperammonemia who were treated with NCG. Their blood ammonia levels were decreased even by the first dose of NCG and normalized in a 6-hour period. In both patients dialysis was avoided. ${ }^{22}$ Also Jones and collaborators published 2 cases of patients with PA and high ammonia levels. The first newborn responded to administration of NCG optimally with a fall in plasma ammonia levels. The second patient did not respond to NCG therapy, but in this case the dose of NCG was lower $(25 \mathrm{mg} / \mathrm{kg}$ rather than $250 \mathrm{mg} / \mathrm{kg}) .{ }^{23}$ Other cases of PA treated with NCG have been described since these cases were described. In these studies the administration of NCG improved detoxification of ammonia. ${ }^{24-27}$ The effectiveness of NCG in decreasing ammonia levels in patients with PA was demonstrated by Tuchman et al. They investigated the in vivo synthesis of $\left[{ }^{13} \mathrm{C}\right]$ urea following administration of $\left[1-{ }^{13} \mathrm{C}\right]$ acetate, a stable isotope tracer, and NCG: in the patient with PA they observed an increase of ${ }^{13} \mathrm{CO}_{2}$ in breath, of $\left[{ }^{13} \mathrm{C}\right]$ urea in plasma and production of absolute $\left[{ }^{13} \mathrm{C}\right]$ urea, although this was lower than in healthy subjects. ${ }^{18}$ Similar results were published by Ah Mew et al: ${ }^{28}$ they administered $\left[{ }^{13} \mathrm{C}\right]$ sodium acetate to 7 patients with PA and neonatal hyperammonemia, and measured the concentration of ${ }^{13} \mathrm{CO}_{2}$ in blood and $\left[{ }^{13} \mathrm{C}\right]$ urea, before and after the start of $\mathrm{NCG}$ therapy. They demonstrated that NCG increases ureagenesis and decreases the blood ammonia and glutamine levels in PA patients.

\section{Methylmalonic acidemia}

Methylmalonic acid is normally derived from propionic acid as part of catabolism of branched-chain amino acids, cholesterol, and odd-chain fatty acids. The enzyme methylmalonyl CoA mutase converts the L-methylmalonic acid to succinic acid. This enzyme requires as a cofactor adenosylcobalamin, a metabolite of vitamin $\mathrm{B}_{12} \cdot{ }^{29}$ Deficiency of enzyme mutase or, less frequently, of adenosylcobalamin determines the accumulation of methylmalonic acid in plasma. Two forms of mutase apoenzyme deficiencies are identified, the mutation $m u t^{0}$ responsible for complete enzyme deficiency and mut responsible for partial enzyme deficiency. ${ }^{30}$ These patients are not responsive to $\mathrm{B}_{12}$-vitamin therapy, unlike the patients with a defect in the formation of adenosylcobalamin. The clinical findings of the patients with MMA due to $m u t^{0}$, mut, and defect in metabolism of vitamin $B_{12}$ are similar. Severe forms develop in the first days of life with feeding problems, vomiting, hypotonia, metabolic acidemia, and consensual tachypnea. Also this condition when not treated promptly may progress to coma and death. The milder forms evolve later with hypotonia, feeding failure, and developmental delay. ${ }^{31}$ Laboratory findings are hyperammonemia and metabolic acidosis with increase of methylmalonic acid in blood. In addition propionic acid and its metabolites are found in the urine. Diagnosis can be confirmed by measuring mutase activity in cultured fibroblasts, or by identifying the mutant gene. Intravenous treatment must be immediately started with carnitine, glucose, insulin, arginine, sodium benzoate, and hypercaloric protein-free diet. It has been demonstrated that the administration of NCG decreases blood ammonia levels. Gebhardt et al described a case of a 6-day-old newborn affected by MMA and hyperammonemia $(805 \mu \mathrm{mol} / \mathrm{L})$. After the initial conventional treatment and peritoneal dialysis his plasma ammonia levels were $610 \mu \mathrm{mol} / \mathrm{L}$. NCG therapy was started and blood ammonia levels dropped to $100 \mu \mathrm{mol} / \mathrm{L}$ 3 hours after the first NCG dose administration. ${ }^{32}$ Also Levrat et al reported a case of MMA in which the hyperammonemia was resolved with insertion of NCG therapy. ${ }^{33}$ 


\section{Maple syrup urine disease}

Branched-chain amino acids (leucine, isoleucine, and valine) are metabolized by a mitochondrial thiamine pyrophosphate (vitamin $\mathrm{B}_{1}$ )-dependent complex enzyme system: branchedchain $\alpha$-ketoacid dehydrogenase. ${ }^{34}$ This enzyme is composed of 3 catalytic components, E1 (decarboxylase component, formed by 2 subunits E1 $\alpha$ and E1 $\beta$ ), E2 (acyltransferase component), and E3 (dehydrogenase component). ${ }^{35}$ Deficiency of this enzyme determines maple syrup urine disease (MSUD, MIM 251000), so called for the typical sweet odor of maple syrup found in body fluids, especially urine. Based on clinical manifestation and response to vitamin $\mathrm{B}_{1}$ administration, 5 types of MSUD have been classified. ${ }^{36}$ All these clinical phenotypes are inherited as an autosomal recessive trait. The gene for $\mathrm{E} 1 \alpha, \mathrm{E} 1 \beta, \mathrm{E} 2$, and $\mathrm{E} 3$ subunits resides on chromosomes $19,6,1$, and 7 , respectively. There is no mutual relationship between clinical phenotypes and genotypes. The classic MSUD is the most severe form with major clinical findings. At birth the newborns are healthy; the clinical manifestations develop in the first week of life. These are poor feeding, vomiting, and in the following days lethargy and coma may appear. These patients are hypertonic, with muscular rigidity and severe opisthotonos. The neurological manifestations are convulsions, hypoglycemia, and cerebral edema may also be present. ${ }^{37}$ In acute state, the neuroimaging may show cerebral edema most frequently in cerebellum, dorsal brainstem, cerebral peduncle, and internal capsule. In later ages of life hypomyelination and cerebral atrophy may be observed. Laboratory findings show increased levels of leucine, isoleucine, and valine in plasma; these amino acids and their respective ketoacids are elevated in urine and in cerebrospinal fluid..$^{38}$ The definitive diagnosis can be done by measuring the enzyme activity in leukocytes or cultured fibroblasts. The treatment of acute state is hydration with glucose infusion and a high caloric protein-free diet. In some patients peritoneal dialysis or hemodialysis are necessary. In 2009 for the first time, Kalkan Ucar et al used NCG in a patient with decompensated MSUD. ${ }^{39}$ These authors demonstrated that the administration NCG together with conventional therapy decreases the blood levels of ammonia and it can be added to improve the acute metabolic decompensation in patients with MSUD.

\section{Isovaleric acidemia}

Isovaleric acidemia (MIM 243500) is a rare autosomal recessive inborn disease caused by deficiency of isovaleryl coenzyme A dehydrogenase (IVD). IVD is a mitochondrial flavoenzyme which catalyzes the conversion of isovaleryl-CoA to 3-methylcrotonyl-CoA in the leucine degradation. Two forms of this disease exist: an acute one in which the symptoms develop in early hours of life with vomiting, severe metabolic acidosis, lethargy, convulsion, and coma, ${ }^{40}$ and a chronic intermittent form defined by episodes of vomiting, dehydration, metabolic acidosis, and alteration of mental status; these events develop in the first months or years of life. Characteristic "sweaty feet" may be present in acute attacks. If untreated, these patients may die or may develop severe central nervous system dysfunctions. Laboratory findings during the acute attacks show metabolic acidosis, neutropenia, and thombocytopenia. Hypocalcemia, hyperglycemia, and moderate to severe hyperammonemia may be present in some patients. ${ }^{41}$ The diagnosis is confirmed by the increase of blood levels of the isovaleric acid and its metabolites and by the elevations of isovalerylglycine and 3-hydroxyisovaleric acid as well as other metabolites in urine. ${ }^{42,43}$ Mass screening in newborn is possible by tandem mass spectrometry (MS/MS) for the analysis of the acylcarnitine profile in blood spot. The gene of IVD has been mapped to chromosome 15q14-15q15. Also it is possible to measure the enzyme activity in leukocytes or cultured fibroblasts. Today many mutations of this gene have been identified. ${ }^{44,45}$ In acute attacks the treatment consists of hydration, correction of metabolic acidosis by infusing sodium bicarbonate, promotion of anabolism by increased caloric intake (infusing glucose), and reduced leucine intake. Treatment must be initiated promptly. The long-term treatment is a diet with lower protein intake $(1.5 \mathrm{~g} / \mathrm{kg} /$ day $)$, and administration of L-carnitine $(100 \mathrm{mg} / \mathrm{kg} /$ day $)$ and glycine $(150-250 \mathrm{mg} / \mathrm{kg} /$ day) to prevent the accumulation of isovaleric acid and its toxic metabolites ${ }^{46}$ Coude et $\mathrm{al}^{47}$ demonstrated in rat hepatocytes that high levels of isovaleric acid inhibited ureagenesis due to a decrease in NAG levels, causing hyperammonemia. For this reason, in patients with isovaleric acidemia associated with hyperammonemia the administration of NCG may be added to conventional therapy. A clinical case report of IVA successfully treated with carglumic acid was recently communicated by Kasapkara et al. ${ }^{48}$

\section{$\mathbf{N}$-carbamylglutamate in hyperinsulinism hyperammonemia syndrome}

The hyperinsulinism hyperammonemia syndrome (HI/ HA) is an inborn error of metabolism. It was described for the first time by Zammarchi et $\mathrm{al}^{49}$ in 1996 . HI/HA syndrome is caused by a mutation of the enzyme glutamate 
dehydrogenase $(\mathrm{GDH})$ encoded by GLUD1. ${ }^{50}$ These mutations result in higher activity of GDH. About $80 \%$ of cases are due to de novo mutations; however a dominant pattern has been demonstrated. ${ }^{51} \mathrm{GDH}$ is a mitochondrial matrix enzyme, expressed at high levels in liver, kidney, brain, and pancreatic $\beta$-cells. It is composed of 6 subunits and oxidizes the glutamate to $\alpha$-ketoglutarate and ammonia using NAD and/or NADP as a cofactor. ${ }^{52}$ The clinical manifestations of affected infants are variable episodes of hypoglycemia associated with hyperammonemia. Hypoglycemia may occur with fasting and in response to protein feeding. ${ }^{53,54}$ Patients with HI/HA syndrome show a large acute insulin response to leucine administration and the blood amino acid concentrations are normal. In these children blood ammonia levels are persistently elevated between 2 and 5 times over normal limit, but the hyperammonemia is not associated with lethargy, irritability, or coma. Stanley has supposed that overactivity of GDH in the brain decreases the levels of glutamate and glutamine, protecting the central nervous system from the neurotoxicity of the accumulation of these elements. ${ }^{55}$ In this metabolic disorder the physiopathological basis of hyperammonemia is that the gain in GDH activity leads to an increase of ammonia levels and excessive oxidation of glutamate reduces the glutamate needed for the synthesis of NAG, slowing the urea cycle. ${ }^{56}$ For hypoglycemia the conventional treatment is diazoxide (5-15 mg/kg/day), a $K_{\text {ATP }}$ channel agonist. Some studies show that NCG is a potential therapy for hyperammonemia. ${ }^{57,58}$

\section{$\mathbf{N}$-carbamylglutamate in valproate- induced hyperammonemia?}

Valproate-induced hyperammonemic encephalopathy is an unusual, but serious, adverse effect of valproic acid treatment that can lead to death, and is characterized by a decreasing level of consciousness, focal neurological deficits, cognitive slowing, vomiting, drowsiness, and lethargy. The mechanism by which hyperammonemia develops is still unclear. Hyperammonemia may result from the stimulation of kidney glutaminase in the renal cortex, from the depletion of mitochondrial acetyl CoA, and decreased production of NAG, but also from the accumulation of propionate, a valproate metabolite. The reduction of hepatic NAG, the obligatory activator of the first enzyme of the urea cycle, leads to the inhibition of hepatic mitochondrial CPS-I. ${ }^{59}$

Two patients with valproate-induced hyperammonemia have been treated with carglumic acid. ${ }^{60} \mathrm{NCG}$ was effective in decreasing blood ammonia levels and its administration may be added to conventional therapy, as suggested by recent guidelines. ${ }^{61}$

\section{Side effects}

The most common adverse reactions (occurring in more than $13 \%$ of patients) observed in patients receiving NCG, regardless of causality, are vomiting, abdominal pain, pyrexia, tonsilitis, anemia, ear infection, diarrhea, nasopharyngitis, and headache. In newborns, NCG is usually well tolerated without significant adverse effects. Tachycardia, profuse sweating, increased bronchial secretion, hyperthermia, and restlessness have been reported only at a dosage of $650 \mathrm{mg} / \mathrm{kg} /$ day. $^{62}$

\section{Conclusion}

We have described the beneficial effects of NCG to decrease blood ammonia levels and to improve ureagenesis in different conditions presenting with hyperammonemia. NCG is safe, fast acting and easy to administer. Dosing varies depending on the clinical condition and indication. Restarting the urea cycle, NCG increases ureagenesis and diminishes blood ammonia levels. It also improves the acute therapy and thus reduces the need for or the duration of peritoneal dialysis and hemodialysis. Favorable long-term outcomes in promptly treated patients with hyperammonemia suggest the need to start the NCG therapy as soon as possible, even before the exact diagnosis is set up. ${ }^{26}$

\section{Abbreviations}

ASL, argininosuccinate lyase; ASS, argininosuccinate synthase; CPS-I, carbamoyl-phosphate synthase I; HI/HA, hyperinsulinism hyperammonemia syndrome; MMA, methylmalonic acidemia; MSUD, maple syrup urine disease; NAG, N-acetylglutamate; NAGS1, N-acetylglutamate synthase; NCG, N-carbamylglutamate; OTC, ornithine transcarbamylase; PA, propionic acidemia.

\section{Disclosure}

The authors report no conflicts of interest in this work.

\section{References}

1. Leonard JV, Ward Platt MP, Morris AA. Hypothesis: proposals for the management of a neonate at risk of hyperammonaemia due to a urea cycle disorder. Eur J Pediatr. 2008;167(3):305-309.

2. O'Connor JE, Jordá A, Grisolía S. Acute and chronic effects of carbamyl glutamate on blood urea and ammonia. Eur J Pediatr. 1985;143(3):196-197.

3. Ah Mew N, Payan I, Daikhin Y, et al. Effects of a single dose of $\mathrm{N}$-carbamylglutamate on the rate of ureagenesis. Mol Gen Metab. 2009; 98(4):325-330. 
4. Kim S, Paik WK, Cohen PP. Ammonia intoxication in rats: protection by L-carbamylglutamate plus L-arginine. Proc Natl Acad Sci U S A. 1972;69(12):3530-3533.

5. Reglero A, Rivas J, Mendelson J, Wallace R, Grisolia S. Deacylation and transacetylation of acetyl glutamate and acetyl ornithine in rat liver. FEBS Lett. 1977;81(1):13-17.

6. Tuchman M, Lee B, Lichter-Konecki U, et al. Cross-sectional multicenter study of patients with urea cycle disorders in the United States. Mol Genet Metab. 2008;94(4):397-402.

7. Bachmann C, Krähenbühl S, Colombo JP, Schubiger G, Jaggi KH, Tönz O. N-acetylglutamate synthetase deficiency: a disorder of ammonia detoxication. $N$ Engl J Med. 1981;304(9):543.

8. Haberle J, Schmidt E, Pauli S, et al. Mutation analysis in patients with N-acetylglutamate synthase deficiency. Hum Mutat. 2003;21(6): 593-597.

9. Caldovic L, Morizono H, Panglao MG, et al. Late onset N-acetylglutamate synthase deficiency caused by hypomorphic alleles. Hum Mutat. 2005; 25(3):293-298.

10. Caldovic L, Morizono H, Tuchman M. Mutations and polymorphisms in the human N-acetylglutamate synthase (NAGS) gene. Hum Mutat. 2007;28(8):754-759.

11. Caldovic L, Ah Mew N, Shi D, Morizono H, Yudkoff M, Tuchman M. $\mathrm{N}$-acetylglutamate synthase: structure, function and defects. Mol Genet Metab. 2010;100 Suppl 1:S13-S19.

12. Gessler P, Buchal P, Schwenk HU, Wermuth B. Favourable long-term outcome after immediate treatment of neonatal hyperammonemia due to N-acetylglutamate synthase deficiency. Eur J Pediatr. 2010;169(2): 197-199.

13. Guffon N, Vianey-Saban C, Bourgeois J, Rabier D, Colombo JP, Guibaud P. A new neonatal case of N-acetylglutamate synthase deficiency treated by carbamylglutamate. J Inherit Metab Dis. 1995; 18(1):61-65.

14. Hinnie J, Colombo JP, Wermuth B, Dryburgh FJ. N-Acetylglutamate synthetase deficiency responding to carbamylglutamate. J Inherit Metab Dis. 1997;20(6):839-840.

15. Morris AA, Richmond SW, Oddie SJ, Pourfarzam M, Worthington V, Leonard JV. N-acetylglutamate synthetase deficiency: favourable experience with carbamylglutamate. J Inherit Metab Dis. 1998;21(8): 867-868.

16. Plecko B, Erwa W, Wermuth B. Partial N-acetylglutamate synthetase deficiency in a 13-year-old girl: diagnosis and response to treatment with N-carbamylglutamate. Eur J Pediatr. 1998;157(12):996-998.

17. Caldovic L, Morizono H, Daikhin Y, et al. Restoration of ureagenesis in $\mathrm{N}$-acetylglutamate synthase deficiency by $\mathrm{N}$-carbamylglutamate. J Pediatr. 2004;145(4):552-554.

18. Tuchman M, Caldovic L, Daikhin Y, et al. N-carbamylglutamate markedly enhances ureagenesis in $\mathrm{N}$-acetylglutamate deficiency and propionic acidemia as measured by isotopic incorporation and blood biomarkers. Pediatr Res. 2008;64(2):213-217.

19. Nordenström A, Halldin M, Hallberg B, Alm J. A trial with $\mathrm{N}$-carbamylglutamate may not detect all patients with NAGS deficiency and neonatal onset. J Inherit Metab Dis. 2007;30(3):400.

20. Kuchler G, Rabier D, Poggi-Travert F, et al. Therapeutic use of carbamylglutamate in the case of carbamoyl-phosphate synthetase deficiency. J Inherit Metab Dis. 1996;19(2):220-222.

21. Coude FX, Sweetman L, Nyhan W. Inhibition by propionyl-coenzyme A of $\mathrm{N}$-acetylglutamate synthetase in rat liver mitochondria. A possible explanation for hyperammonemia in propionic and methylmalonic acidemia. J Clin Invest. 1979;64(6):1544-1551.

22. Gebhardt B, Dittrich S, Parbel S, Vlaho S, Matsika O, Bohles H. $\mathrm{N}$-carbamylglutamate protects patients with decompensated propionic aciduria from hyperammonaemia. J Inherit Metab Dis. 2005;28(2): 241-244.

23. Jones S, Reed CA, Vijay S, Walter JH, Morris AA. N-Carbamylglutamate for neonatal hyperammonaemia in propionic acidaemia. J Inherit Metab Dis. 2008 Feb 21. [Epub ahead of print].
24. Fernández de Miguel S, Gimeno Díaz de Atauri A, Torres Peral R, Fernández Carrión F, Serrano Ayestarán O. N-carbamyl glutamate treatment in hyperammoniemia decompensated propionic acidaemia. An Pediatr (Barc). 2009;71(6):579-580.

25. Schwahn BC, Pieterse L, Bisset WM, Galloway PG, Robinson PH. Biochemical efficacy of N-carbamylglutamate in neonatal severe hyperammonaemia due to propionic acidaemia. Eur J Pediatr. 2010; 169(1):133-134.

26. Filippi L, Gozzini E, Fiorini P, Malvagia S, la Marca G, Donati MA. $\mathrm{N}$-carbamylglutamate in emergency management of hyperammonemia in neonatal acute onset propionic and methylmalonic aciduria. Neonatology. 2010;97(3):286-290.

27. Soyucen E, Demirci E, Aydin A. Outpatient treatment of propionic acidemia-associated hyperammonemia with N-carbamoyl-L-glutamate in an infant. Clin Ther. 2010;32(4):710-713.

28. Ah Mew N, McCarter R, Daikhin Y, Nissim I, Yudkoff M, Tuchman M. N-carbamylglutamate augments ureagenesis and reduces ammonia and glutamine in propionic acidemia. Pediatrics. 2010;126(1): e208-e214.

29. Acquaviva C, Benoist JF, Pereira S, et al. Molecular basis of methylmalonyl-CoA mutase apoenzyme defect in 40 European patients affected by mut(o) and mut- forms of methylmalonic acidemia: identification of 29 novel mutations in the MUT gene. Hum Mutat. 2005;25(2):167-176.

30. Fowler B, Leonard JV, Baumgartner MR. Causes of and diagnostic approach to methylmalonic acidurias. J Inherit Metab Dis. 2008;31(3): 350-360.

31. Manoli I, Venditti CP. Methylmalonic acidemia. In: Pagon RA, Bird TC, Dolan CR, Stephens K, editors. GeneReviews. Seattle (WA): University of Washington; 1993-2005.

32. Gebhardt B, Vlaho S, Fischer D, SewellA, Böhles H. N-carbamylglutamate enhances ammonia detoxification in a patient with decompensated methylmalonic aciduria. Mol Genet Metab. 2003;79(4):303-304.

33. Levrat V, Forest I, Fouilhoux A, Acquaviva C, Vianey-Saban C, Guffon N. Carglumic acid: an additional therapy in the treatment of organic acidurias with hyperammonemia? Orphanet J Rare Dis. 2008;3:2.

34. Dancis J, Hutzler J, Levitz M. Metabolism of the white blood cells in maple-syrup-urine disease. Biochim Biophys Acta. 1960;43:342-343.

35. Chuang DT. Maple syrup urine disease: it has come a long way. J Pediatr. 1998;132(3 Pt 2):S17-S23.

36. Chuang DT, Shih VE. Disorders of branched-chain amino acid and keto acid metabolism. In: Scriver CR, Beaudet AL, Sly WS, Valle D, editors. The Metabolic and Molecular Basis of Inherited Disease. 7th ed. New York: McGraw-Hill; 1995:1239-1277.

37. Crome L, Dutton G, Ross CF. Maple syrup urine disease. J Pathol Bacteriol. 1961;81:379-384.

38. Mackenzie DY, Woolf LI. Maple syrup urine disease; an inborn error of the metabolism of valine, leucine, and isoleucine associated with gross mental deficiency. Br Med J. 1959;1(5114):90-91.

39. Kalkan Ucar S, Coker M, Habif S, et al. The first use of $\mathrm{N}$-carbamylglutamate in a patient with decompensated maple syrup urine disease. Metab Brain Dis. 2009;24(3):409-414.

40. Lott IT, Erickson AM, Levy HL. Dietary treatment of an infant with isovaleric acidemia. Pediatrics. 1972;49(4):616-618.

41. Mendiola J Jr, Robotham JL, Liehr JG, Williams JC. Neonatal lethargy due to isovaleric acidemia and hyperammonemia. Tex Med. 1984;80(1): 52-54.

42. Tanaka K, Budd MA, Efron ML, Isselbacher KJ. Isovaleric acidemia: a new genetic defect of leucine metabolism. Proc Natl Acad Sci US A. 1966;56(1):236-342.

43. Tanaka K, Isselbacher KJ. The isolation and identification of $\mathrm{N}$-isovalerylglycine from urine of patients with isovaleric acidemia. J Biol Chem. 1967;242(12):2966-2972.

44. Vockley J, Rogan PK, Anderson BD, et al. Exon skipping in IVD RNA processing in isovaleric acidemia caused by point mutations in the coding region of the IVD gene. Am J Hum Genet. 2000;66(2):356-367. 
45. Lee YW, Lee DH, Vockley J, Kim ND, Lee YK, Ki CS. Different spectrum of mutations of isovaleryl-CoA dehydrogenase (IVD) gene in Korean patients with isovaleric acidemia. Mol Genet Metab. 2007; 92(1-2):71-77.

46. Vockley J, Ensenauer R. Isovaleric acidemia: new aspects of genetic and phenotypic heterogeneity. Am J Med Genet C Semin Med Genet. 2006;142C(2):95-103.

47. Coude FX, Grimber G, Parvy P, Rabier D. Role of N-acetylglutamate and acetyl-CoA in the inhibition of ureagenesis by isovaleric acid in isolated rat hepatocytes. Biochim Biophys Acta. 1983;761(1):13-16.

48. Kasapkara CS, Ezgu FS, Tumer L, Biberoglu G, Okur I, Hasanoglu A. $\mathrm{N}$-carbamylglutamate treatment for acute neonatal hyperammonaemia in isovaleric acidaemia. J Inherit Metab Dis. 2010;33 Suppl 1:S48.

49. Zammarchi E, Filippi L, Novembre E, Donati MA. Biochemical evaluation of a patient with a familial form of leucine-sensitive hypoglycemia and concomitant hyperammonemia. Metabolism. 1996; 45(8):957-960.

50. Stanley CA, Lieu YK, Hsu BY, et al. Hyperinsulinism and hyperammonemia in infants with regulatory mutations of the glutamate dehydrogenase gene. N Engl J Med. 1998;338(19):1352-1357.

51. MacMullen C, Fang J, Hsu BY, et al. Hyperinsulinism/hyperammonemia syndrome in children with regulatory mutations in the inhibitory guanosine triphosphate-binding domain of glutamate dehydrogenase. J Clin Endocrinol Metab. 2001;86(4):1782-1787.

52. Fahien LA, MacDonald MJ, Kmiotek EH, Mertz RJ, Fahien CM. Regulation of insulin release by factors that also modify glutamate dehydrogenase. J Biol Chem. 1988;263(27):13610-13614.

53. Parini R, Colombo F, Lombardi AM, Menni F, Beccaria L. Hyperinsulinism plus hyperammonemia. J Pediatr. 1998;133(6):800-801.

54. Hsu BY, Kelly A, Thornton PS, Greenberg CR, Dilling LA, Stanley CA. Protein-sensitive and fasting hypoglycemia in children with the hyperinsulinism/hyperammonemia syndrome. J Pediatr. 2001; 138(3):383-389.
55. Stanley CA. Hyperinsulinism/hyperammonemia syndrome: insights into the regulatory role of glutamate dehydrogenase in ammonia metabolism. Mol Genet Metab. 2004;81 Suppl 1:S45-S51.

56. Huijmans JG, Duran M, de Klerk JB, Rovers MJ, Scholte HR. Functional hyperactivity of hepatic glutamate dehydrogenase as a cause of the hyperinsulinism/hyperammonemia syndrome: effect of treatment. Pediatrics. 2000;106(3):596-600.

57. Palladino AA, Stanley CA. The hyperinsulinism/hyperammonemia syndrome. Rev Endocr Metab Disord. 2010 Oct 9. [Epub ahead of print].

58. De Lonlay P, Benelli C, Fouque F, et al. Hyperinsulinism and hyperammonemia syndrome: report of twelve unrelated patients. Pediatr Res. 2001;50(3):353-357.

59. Segura-Bruna N, Rodriguez-Campello A, Puente V, Roquer J. Valproate-induced hyperammonemic encephalopathy. Acta Neurol Scand. 2006;114(1):1-7.

60. Pedron Giner C, Lopez Marin L, Quijada Fraile P, et al. Valproate induced hyperammonaemic encephalopathy syndrome. Treatment with carglumic acid. J Inherit Metab Dis. 2008;31 Suppl 1:S89.

61. Protocolo Hispano-Luso de diagnostico y tratamiento de las hiperamoniemias en pacientes neonatos y de màs de 30 dias de vida. 2nd ed. Ergon ed. Majadahonda, Madrid; 2009.

62. Schubiger G, Bachmann C, Barben P, Colombo JP, Tönz O, Schüpbach D. N-acetylglutamate synthetase deficiency: diagnosis, management and follow-up of a rare disorder of ammonia detoxication. Eur J Pediatr. 1991;150(5):353-356.
International Journal of General Medicine

\section{Publish your work in this journal}

The International Journal of General Medicine is an international, peer-reviewed open-access journal that focuses on general and internal medicine, pathogenesis, epidemiology, diagnosis, monitoring and treatment protocols. The journal is characterized by the rapid reporting of reviews, original research and clinical studies across all disease areas.

\section{Dovepress}

A key focus is the elucidation of disease processes and management protocols resulting in improved outcomes for the patient.The manuscript management system is completely online and includes a very quick and fair peer-review system. Visit http://www.dovepress.com/ testimonials.php to read real quotes from published authors. 